\title{
LARGE-SCALE GALACTIC AND INTERGALACTIC MAGNETIC FIELDS
}

\author{
M. FUJIMOTO ${ }^{1}$ and T. SAWA ${ }^{2}$ \\ 1 Department of Physics, Nagoya University, \\ Nagoya 464-01, Japan \\ 2 Department of Earth Sciences, Aichi University of Education, \\ Kariya, Aichi 448, Japan
}

\begin{abstract}
Large-scale axisymmetric and bisymmetric spiral (ASS and BSS) structures are found of magnetic fields in spiral galaxies by measuring the Faraday rotation of polarized radio emission. Dynamo theory is introduced to explain the field structures, and strong magnetogravitational interaction is suggested to occur between the BSS magnetic fields and spiral density waves. Up-to-date data about the rotation measures $R M$ and redshifts $z$ of QSOs and distant radio galaxies are given for discussing large-scale intergalactic magnetic fields.
\end{abstract}

\section{Large-Scale Galactic Magnetic Fields}

According to high-resolution radio observations of the spiral M51 (Segalovitz et al. 1976), polarization planes at $\lambda=6$ and $21 \mathrm{~cm}$ are distributed smoothly over the disk. Tosa and Fujimoto (1978) developed a technique to discriminate between the ASS and BSS magnetic field structures (figure 1). Figure 2 shows a first application of this method for the BSS

(a)
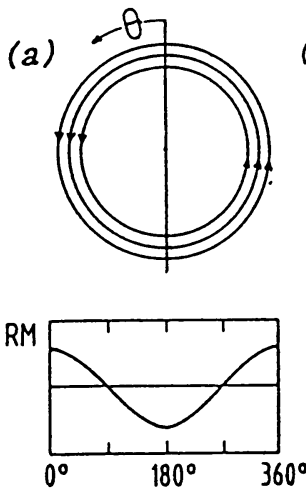

(b)
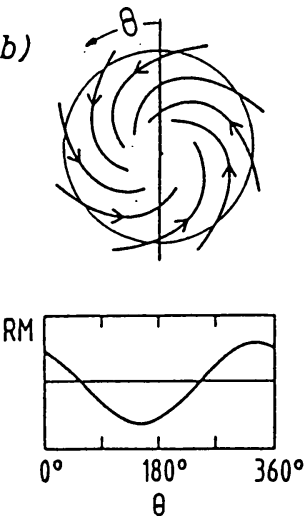

(c)
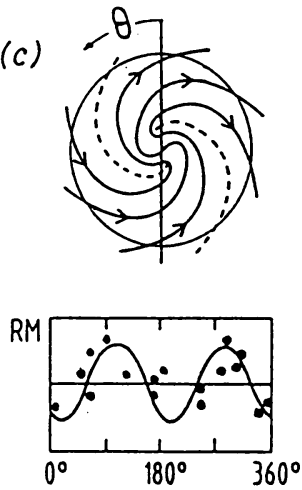

Fig.1. Ring (a), axisymmetric spiral (b), and bisymmetric spiral (c) configurations of the galactic magnetic fields, and the corresponding single, single and phase-shifted, and double periodic distributions of the Faraday rotation measure $R M$ along the azimuthal angle $\theta$ in the disk. The straight lines denote the lines of node, from which the angle $\theta$ is measured. The left-hand side of each galaxy is toward us. The dotted data in the right bottom diagram are reproduced from Tosa and Fujimoto (1978) for obtaining a picture of the BSS magnetic fields in M51 in figure 2. 
magnetic fields in spiral galaxies. Sofue, Takano and Fujimoto at Nagoya, and Beck, Krause, Gräve, Buczilowski, Hummel, Klein, and Wielebinski at Bonn have made extensive observations and similar analyses of radio polarization of other spiral galaxies. For summary of the data see Sofue, Fujimoto and Wielebinski (1986), Beck (1989), and Krause (1989). Two to four spirals [M33(?), M51, M81, and probably the Galaxy] have the BSS magnetic fields and two spirals (M31 and IC342) the ASS ones. Other several spirals M83, M101, NGC4258, NGC6946 etc. have large-scale mean fields, but their global configurations, ASS or BSS, are not conclusive yet. Some number of spirals seem to have the BSS magnetic fields.

\section{The BSS Magnetic Field in the Galaxy}

Simard-Normandin and Kronberg (1980) are the first who used the observed relation between $R M \mathrm{~s}$ and Galactic longitudes $l$ of extragalactic radio sources and indicated sudden reversals of magnetic fields interior to the solar circle of the Galactic plane. The same field reversal is confirmed to occur at $\sim 170 \mathrm{pc}$ from the Sun toward the Galactic center, based on both rotation and dispersion measures of pulsars (Thomson and Nelson 1980). These results and recent analyses by Sofue and Fujimoto (1983) and Vallee (1988) give us a picture that is consistent with the BSS magnetic fields along the spiral arms (figure 3 ).

3. Theories for Galactic Magnetic Fields and their Interaction with Spiral Density Waves

Concentric ring fields in galaxies are free from the twisting due to differential rotation. The alignment of magnetic fields along the spiral arms would be due to local compression of magnetized gas in the galactic shock waves (Roberts and Yuan 1970, Tosa 1973). The existence of the BSS magnetic fields requires us, however, their quick diffusion and regeneration in the disk, otherwise the BSS fields are too tightly twisted by differential rotation in some hundred million years. Sawa and Fujimoto (1986) and Baryshnikova et al. (1987) introduced a nonaxisymmetric dynamo theory in the galactic disk and showed that the mildly-spiral field pattern is maintained in a steady state through the process that the tightly-twisted (toroidal) component diffuses away on turbulent motion of interstellar gas and then poloidal component is generated by

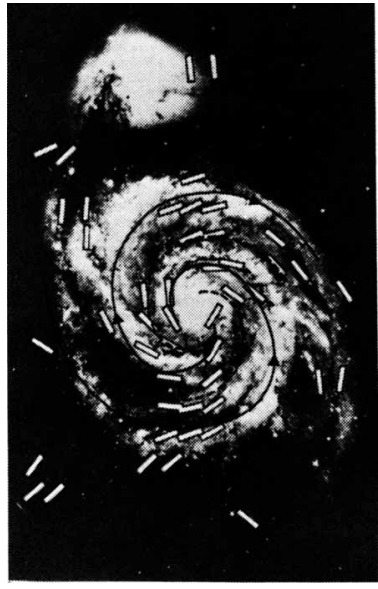

Fig. 2. Orientations of magnetic lines of force projected onto the disk of M51. Photo is due to A. Sandage (the Carnegie Institution of Washignton, 1961). The BSS structures are determined from the double-periodic distribution of $R M$ along the azimuthal angle $\theta$ in figure 1. The line of node is approximately parallel to the NorthSouth line and the east side is toward us.

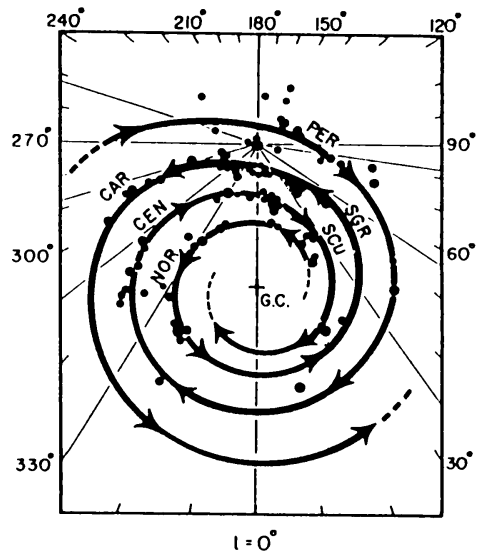

Fig. 3. The BSS magnetic fields in the Galaxy (reproduced from Sofue and Fujimoto 1983). 
cyclonic turbulence and twisted by differential rotation (see also Fujimoto 1987 and Ruzmaikin 1987). It is found that the BSS field pattern makes more easily a solid-body rotation as a wave over the galactic plane, if the dynamo strength $\alpha$ is negative and the disk thickness is a few times as large as that of neutral hydrogen layer (Sawa and Fujimoto 1987). The dynamo equation they employed is,

$$
\frac{\partial \boldsymbol{B}}{\partial t}=\operatorname{rot}(\boldsymbol{V} \times \boldsymbol{B})+\eta \nabla^{2} \boldsymbol{B}+\operatorname{rot}(\alpha \boldsymbol{B}),
$$

with $\operatorname{div} B=0$, where $\eta$ is the turbulent diffusion constant of magnetic fields. An asymptotic solution in the following form is searched for,

$$
\boldsymbol{B}=\boldsymbol{b}(r) \exp \left[m i\left(-\omega t+k z+\phi+\frac{1}{\epsilon} \ln r\right)\right],
$$

where the amplitude $b(r)$ is a slow function of $r$, and $\epsilon$ is a small number $(\sim 0.1)$ so chosen as to reproduce the observed logarithmic spirals. When $m=1$, the field distribution in equation (2) is bisymmetric. Substituting equation (2) into (1) and taking dominant terms after series expansion in $\epsilon(\ll 1)$, we have a dispersion relation for $\omega$ and $k$, in which $\operatorname{Re}(\omega)$ is the angular velocity of the BSS magnetic field pattern. Theoretical field distributions thus obtained are given in figures 1 and 2 of Sawa and Fujimoto (1989) in this volume.

We note that the BSS magnetic field and the spiral arm are entirely different waves but their pattern angular velocities, $\operatorname{Re}(\omega)$ and $\Omega_{p}$, are numerically close to each other and thus a strong resonance occurs between them enhancing the dynamo action and spiral condensation of gas (Fujimoto and Sawa 1989, Chiba and Tosa 1989). The BSS magnetic fields could compensate the spiral density waves for their secular dispersion (Toomre 1969).

\section{Large-Scale Cosmic Magnetic Fields}

The BSS magnetic fields in figures 2 and 3 are topologically not inconsistent with uniform cosmic magnetic fields trapped to protogalaxies, twisted by differential rotation and maintained till now by the dynamo action. Sofue, Fujimoto and Kawabata (1968) claimed a detection of intergalactic magnetic field based on measurements of the Faraday rotation

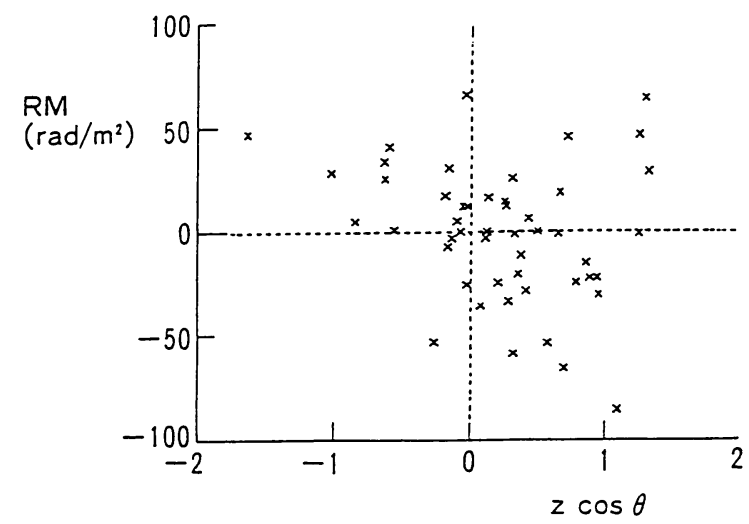

Fig. 4. The $R M-z \cos \theta$ relation for the radio active QSOs at high galactic latitudes $|b|>35^{\circ}$ and with large redshifts $z>1$. 
measures of QSOs and distant radio galaxies in higher galactic latitudes $|b|>35^{\circ}$. The mean field is uniform in the space up to the redshift of $z \sim 1$ and its strength is of order $10^{-9}$ gauss. Although their extensive data of $R M$ and $z$ obtained and compiled by Tabara and Inoue (1989) and Tabara (1989) have smeared our earlier $R M-z \cos \theta$ relation of QSOs and radio galaxies of $z \leq 1$, the most recent analysis recovers this relation for QSOs of $z>1$ and with reliable $R M$, and as figure 4 shows Tabara and Fujimoto (1989) suggest again the existence of large-scale cosmic magnetic field in space of $z>1$, where $\theta$ 's are the angles between the QSOs of $z>1$ and a direction of cosmic magnetic field $\left(l \simeq 110^{\circ}, b \simeq 15^{\circ}\right)$.

Kronberg and Perry (1982) and Welter, Perry, and Kronberg (1984) have discovered that the rotation measures are systematically larger for polarized radio waves passing through clusters of galaxies or/and intervening intergalactic gas clouds than for those not passing through such foreground gaseous objects. The field strength is $2 \times 10^{-6}$ gauss and scale length is several tens of kpc. They are magnetized gaseous halos of distant galaxies and extended gas in clusters of galaxies. In any way, some direct evidence has been obtained for the presence of magnetic fields in diffuse gas at cosmological distances.

\section{References}

Baryshnikova, Y., Ruzmaikin, A., Sokoloff IV, D.D., and Shukurov, A. (1987) Astron. Astrophys. 177, 27-41.

Beck, R. (1989) Geophysical and Astrophysical Fluid Dynamics (special issue), in press.

Chiba, M. and Tosa, M. (1989) submitted to Mon. Not. R. astr. Soc.

Fujimoto, M. (1987) R. Beck and R. Gräve (eds), Interstellar Magnetic Fields, Springer Verlag, Berlin, 23-29.

Fujimoto, M. and Sawa, T. (1989) Geophysical and Astrophysical Fluid Dynamics (special issue), in press.

Krause, M. (1989) This Volume.

Kronberg, P.P. and Perry, J.J. (1982) Astrophys. J. 263, 518-532.

Roberts, W.W. and Yuan, C. (1970) Astrophys. J. 161, 887-902.

Ruzmaikin, A. (1987) R. Beck and R. Gräve (eds), Interstellar Magnetic Fields, Springer Verlag, Berlin, 16-22.

Sawa, T. and Fujimoto, M. (1986) Publ. Astron. Soc. Japan 38, 133-150.

Sawa, T. and Fujimoto, M. (1987) E. Asséo and D. Grésillon (eds), Magnetic Fields and Extragalactic Objects , Editions de Physique (BP112, 91944 Les Ulis Cedex, France), 165-169.

Sawa, T. and Fujimoto, M. (1989) This Volume.

Segalovitz, A., Shane, W.W., and de Bruyn, A.G. (1976) Nature 264, 222-226.

Simard-Normandin, M. and Kronberg, P.P. (1980) Astrophys. J. 242, 74-94.

Sofue, Y. and Fujimoto, M. (1983) Astrophys. J. 265, 722-729.

Sofue, Y., Fujimoto, M., and Kawabata, K. (1968) Publ. Astron. Soc. Japan 20, pp. 388-395.

Sofue, Y., Fujimoto, M., and Wielebinski, R. (1986) Ann. Rev. Astron. Astrophys. 24, 459-497.

Tabara, H. (1989) private communication.

Tabara, H. and Inoue, M. (1980) Astron. Astrophys. Suppl. 39, 379-393.

Tabara, H. and Fujimoto, M. (1989) in preparation.

Thomson, R.C. and Nelson, A.H. (1980) Mon. Not. R. astr. Soc. 191, 863-870.

Toomre, A. (1969) Astrophys. J. 158, 899-913.

Tosa, M. (1973) Publ. Astron. Soc. Japan 25, 191-205.

Tosa, M. and Fujimoto, M. (1978) Publ. Astron. Soc. Japan 30, 315-325.

Vallée, J.P. (1988) Astron. J. 95, 750-754.

Welter, G.L., Perry, J.J. and Kronberg, P.P. (1984) Astrophys. J. 279, 19-39. 
F. KRAUSE: You talked about interaction between gravity waves and magnetic field modes. Since the main mass in the gravity waves is found in stars and stellar objects I cannot understand in which way the magnetic field is able to influence it.

FUJIMOTO: When the disk of gas and stars is marginal or slightly unstable against spiral perturbation, the Lorentz force due to the BSS fields contributes to the spiral condensation of the stellar component through the gravitational force of the gaseous arms. According to Toomre the spiral density waves disperse in $\sim 10^{9}$ years in the Galaxy. If the disk remains marginal or/and slightly unstable after the spiral density waves disperse away, the BSS magnetic fields work again to generate and enhance the spiral condensations of gas and stellar components.

When the density of gas is $\sim 1 / 10$ of that of the stellar component, for example, the spiral density wave can be maintained if the spiral gas doubles its density in $3.10^{8}$ years. This process works if the angular velocities of the BSS fields and spiral density waves are sufficiently equal to each other. However, if these two angular velocities are numerically different and the BSS fields are weak, this process does not work, and, as you questioned, the BSS fields do not contribute to the maintenance of the stellar density waves.

SHUKUROV: The idea that bisymmetric magnetic fields can serve as a trigger of spiral patterns seems to be very fruitful although not appreciated in full by specialists in spiral pattern theory. Prof. Fujimoto has investigated some aspects of this problem and reached encouraging conclusions.

BECK: The occurrence of reversals does not prove the existence of a bisymmetric field, as the dynamo may excite axisymmetric field tori with alternating directions.

SHUKUROV: In your dynamo model $\alpha\langle 0$ for $z>0$. In other dynamo models developed for disk geometry the field decays in this situation (for $d \Omega / d r<0)$. Why is your model different from other ones?

FUJIMOTO: The dynamo action of $\alpha \geqslant 0$ in $z \geqslant 0$ generates new poloidal fields which are twisted by differential rotation to diminish the original fields. In this sense, your comments are correct, but the azimuthally periodic field pattern can rotate with high phase velocity. The decay of the field is delayed by a thicker disk whose scale height is two or four times as large as the neutral hydrogen layer.

KAHN: Quasar absorption lines are formed in galactic halos. The observation of Faraday rotation provides evidence for magnetic fields in galactic halos. 